\title{
ITERATIVE ALGORITHM FOR THE DESIGN OF OPTIMAL FIR ANALYSIS/SYNTHESIS FILTERS FOR OVERDECIMATED FILTER BANKS
}

\author{
Andre Tkacenko and P. P. Vaidyanathan \\ Dept. of Electrical Engineering \\ California Institute of Technology, Pasadena, CA 91125, USA \\ E-mail: andre@systems.caltech.edu,ppvnath@systems.caltech.edu
}

\begin{abstract}
Recently, much attention has been given to the design of signaladapted filter banks, in which the filter banks are designed to optimize a particular objective function, i.e. coding gain or a multiresolution criterion, for a particular class of input signals. If we restrict the analysis/synthesis filters to satisfy an orthonormality or biorthogonality condition, but put no restrictions on filter orders, then often times it is known how to choose the filters optimally for the objectives mentioned above. However, such filters are often unrealizable infinite order filters. In this paper, we consider the design of optimal analysis/synthesis filters in which the only restriction is that they must be finite impulse response (FIR) filters. We focus here on minimizing the mean squared reconstruction error for overdecimated filter banks. An iterative method to alternately design the analysis and synthesis banks is presented in which the error is monotonic nonincreasing for each iteration. Simulation results provided show the merit of the proposed algorithm. ${ }^{1}$
\end{abstract}

\section{INTRODUCTION}

In the past few years, the design of signal-adapted filter banks has attracted the attention of the signal processing and data compression communities $[5,9,4]$. Such filter banks are designed to optimize a particular objective, i.e. coding gain or a multiresolution criterion, adapted for a specific class of input signals. For simplicity, the analysis and synthesis filters are often chosen to satisfy an orthonormality or biorthogonality condition. If no other restrictions are put on the filters, then the optimal choice for these filters is known (see $[5,8,1]$ for the orthonormal case and $[6,9,4]$ for the biorthogonal case). In the orthonormal case, the optimal solution is an infinite order principal component filter bank (PCFB) $[8,1]$, whereas in the biorthogonal case, the optimal filter bank is a PCFB with a parallel bank of half-whitening filters in the middle of the system $[9,4]$. Such filter banks, be they restricted to being orthonormal or biorthogonal, are optimal for a wide variety of rate-distortion criteria including coding gain and reconstruction error if only a subset of subbands is preserved (i.e. multiresolution criterion) $[1,4]$. The only problem is that such optimal filter banks require ideal analysis/synthesis filters and are as such unrealizable.

If we restrict the analysis/synthesis filters to be finite impulse response (FIR) filters, then in general there is no closed form expression for an optimal signal-adapted filter bank and numerical

\footnotetext{
${ }^{1}$ Work supported in part by the ONR grant N00014-99-1-1002, USA.
}

techniques must be employed. Furthermore, such filter banks are only optimal for one specific objective, unlike unconstrained order filter banks which are simultaneously optimal for a wide variety of objectives. For the orthonormal case, in [3] the authors considered a multiresolution optimality criterion, whereas in [10], the authors considered optimizing coding gain. In [4], the authors considered optimizing a particular rate-distortion function for the more general biorthogonal case. For all such cases, complicated nonlinear optimization techniques were required.

In this paper, we focus on the design of optimal signal-adapted filter banks in which the analysis/synthesis filters are constrained to be FIR, but with no other constraints made on the filters. An iterative algorithm for finding the optimum filter bank, in which the analysis and synthesis banks are alternately optimized, is proposed. The objective we focus on here is the mean squared reconstruction error obtained when only a subset of the subbands of a maximally decimated system [7] are kept. At each stage of the iteration, the optimal analysis/synthesis bank can be computed in closed form using the principle of least squares. As a result, the error at each iteration is guaranteed to not increase. Simulation results provided verify this monotonic behavior.

\subsection{Overdecimated Filter Bank Signal Model}

For the reconstruction error objective mentioned above, we focus here on the overdecimated uniform filter bank shown in Fig. 1(a). By overdecimated, we mean that the number of channels $L$ satisfies $L<M$, i.e. the number of subbands is strictly less than the decimation ratio [7]. In such a system, there is always necessarily a loss of information and in general, alias cancellation and perfect reconstruction are impossible. If we consider the following polyphase decompositions [7] of the analysis filters $H_{k}(z)$ and synthesis filters $F_{k}(z)$ for $0 \leq k \leq L-1$,

$$
\begin{aligned}
& H_{k}(z)=\sum_{\ell=0}^{M-1} z^{\ell} H_{k, \ell}\left(z^{M}\right) \quad \text { (Type II) } \\
& F_{k}(z)=\sum_{\ell=0}^{M-1} z^{-\ell} F_{k, \ell}\left(z^{M}\right) \quad \text { (Type I) }
\end{aligned}
$$

then the system of Fig. 1(a) can be redrawn as in Fig. 1(b), where,

$$
[\mathbf{H}(z)]_{\ell, m}=H_{\ell, m}(z),[\mathbf{F}(z)]_{m, \ell}=F_{\ell, m}(z)
$$

for $0 \leq \ell \leq L-1$ and $0 \leq m \leq M-1$. Note that here, the vector signals $\mathbf{x}(n)$ and $\mathbf{y}(n)$ denote, respectively, the $M$-fold blocked versions [7] of the filter bank input $x(n)$ and output $y(n)$. 


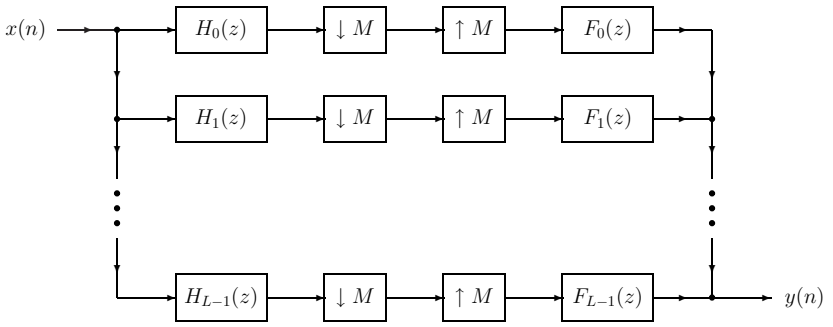

(a)

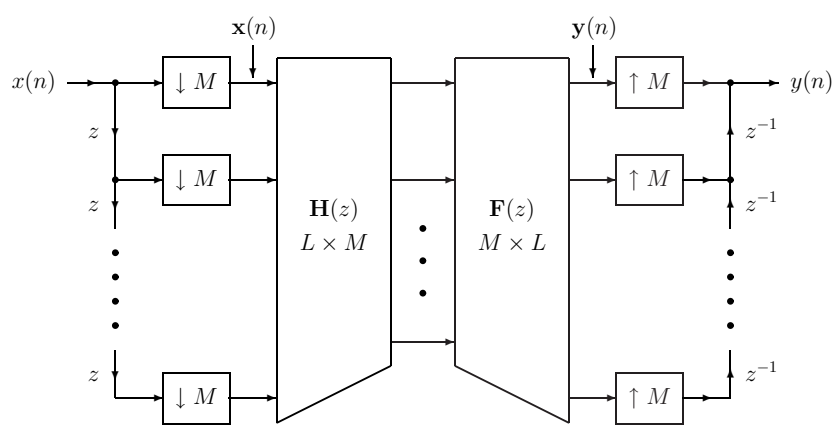

(b)

Fig. 1. (a) Uniform overdecimated filter bank $(L<M)$, (b) polyphase representation.

\section{OPTIMIZING THE RECONSTRUCTION ERROR}

As mentioned earlier, in general, perfect reconstruction is not possible for the overdecimated filter bank system of Fig. 1. The question then arises as to how to best choose the analysis and synthesis polyphase matrices $\mathbf{H}(z)$ and $\mathbf{F}(z)$, subject to an FIR constraint, such that the error in reconstruction is minimized. Here, we will assume that the input $x(n)$ is a cyclo wide sense stationary process of period $M(\mathrm{CWSS}(M))$ [7], which is equivalent to saying that its $M$-fold blocked version $\mathbf{x}(n)$ is wide sense stationary (WSS). Then, the $M$-fold blocked version of the output $y(n)$, namely $\mathbf{y}(n)$, is jointly WSS with $\mathbf{x}(n)$. The objective that we seek to minimize is the expected mean squared reconstruction error between $\mathbf{x}(n)$ and $\mathbf{y}(n)$, defined as follows.

$$
\xi \triangleq E\left[\|\mathbf{x}(n)-\mathbf{y}(n)\|^{2}\right]
$$

If we define the blocked filter bank error $\mathbf{e}(n)$ as $\mathbf{e}(n) \triangleq \mathbf{x}(n)-$ $\mathbf{y}(n)$ and denote the power spectral densities of $\mathbf{x}(n)$ and $\mathbf{e}(n)$ to be $\mathbf{S}_{\mathbf{x x}}(z)$ and $\mathbf{S}_{\mathbf{e e}}(z)$, respectively, then from (1), we have,

$$
\xi=\operatorname{Tr}\left[\frac{1}{2 \pi} \int_{0}^{2 \pi} \mathbf{S}_{\mathbf{e e}}\left(e^{j \omega}\right) d \omega\right]
$$

where $\mathbf{S}_{\mathbf{e e}}(z)$ is given by Fig. 1(b) and [7] to be,

$$
\begin{aligned}
\mathbf{S}_{\mathbf{e e}}(z)= & \mathbf{S}_{\mathbf{x x}}(z)-\mathbf{F}(z) \mathbf{H}(z) \mathbf{S}_{\mathbf{x x}}(z)-\mathbf{S}_{\mathbf{x x}}(z) \widetilde{\mathbf{H}}(z) \widetilde{\mathbf{F}}(z) \\
& +\mathbf{F}(z) \mathbf{H}(z) \mathbf{S}_{\mathbf{x x}}(z) \widetilde{\mathbf{H}}(z) \widetilde{\mathbf{F}}(z)
\end{aligned}
$$

Here $\widetilde{\mathbf{G}}(z) \triangleq \mathbf{G}^{\dagger}\left(1 / z^{*}\right)$ for any $\mathbf{G}(z)$ [7].

We will now derive the optimal synthesis/analysis filter bank, subject to an FIR constraint, for a fixed analysis/synthesis system.

\subsection{Optimal Synthesis Bank For Fixed Analysis Bank}

Suppose that the analysis bank $\mathbf{H}(z)$ is fixed and that the synthesis bank $\mathbf{F}(z)$ is FIR of length $N_{f}$ and of the form,

$$
\mathbf{F}(z)=z^{P} \mathbf{F}_{c}(z)
$$

where $P$ is an advance parameter and $\mathbf{F}_{c}(z)$ is a causal FIR system of the form,

$$
\mathbf{F}_{c}(z)=\sum_{n=0}^{N_{f}-1} \mathbf{f}_{c}(n) z^{-n}
$$

Note that the impulse response $\mathbf{f}_{c}(n)$ is an $M \times L$ sequence. If we define the $M \times L N_{f}$ matrix $\overline{\mathbf{f}}_{c}$ and $L N_{f} \times L$ delay matrix $\mathbf{d}(z)$ as,

$$
\begin{aligned}
\overline{\mathbf{f}}_{c} & \triangleq\left[\begin{array}{llll}
\mathbf{f}_{c}(0) & \mathbf{f}_{c}(1) & \cdots & \mathbf{f}_{c}\left(N_{f}-1\right)
\end{array}\right] \\
\mathbf{d}(z) & \triangleq\left[\begin{array}{lllll}
z^{P} \mathbf{I}_{L} & z^{P-1} \mathbf{I}_{L} & \cdots & z^{P-\left(N_{f}-1\right)} \mathbf{I}_{L}
\end{array}\right]^{T}
\end{aligned}
$$

then clearly we have $\mathbf{F}(z)=\overline{\mathbf{f}}_{c} \mathbf{d}(z)$ and all of the degrees of freedom in choosing $\mathbf{F}(z)$ with the FIR constraint lie in the choice of the constant matrix $\overline{\mathbf{f}}_{c}$. From (2) and (3), we have,

$$
\xi=\operatorname{Tr}\left[\mathbf{R}_{\mathbf{x x}}(0)-\mathbf{B}^{\dagger} \overline{\mathbf{f}}_{c}^{\dagger}-\overline{\mathbf{f}}_{c} \mathbf{B}+\overline{\mathbf{f}}_{c} \mathbf{A} \overline{\mathbf{f}}_{c}^{\dagger}\right]
$$

where $\mathbf{R}_{\mathbf{x x}}(k)$ denotes the autocorrelation sequence of $\mathbf{x}(n)$ and the $L N_{f} \times L N_{f}$ matrix $\mathbf{A}$ and $L N_{f} \times M$ matrix $\mathbf{B}$ are defined as,

$$
\begin{gathered}
\mathbf{A} \triangleq \frac{1}{2 \pi} \int_{0}^{2 \pi} \mathbf{d}\left(e^{j \omega}\right) \mathbf{H}\left(e^{j \omega}\right) \mathbf{S}_{\mathbf{x x}}\left(e^{j \omega}\right) \mathbf{H}^{\dagger}\left(e^{j \omega}\right) \mathbf{d}^{\dagger}\left(e^{j \omega}\right) d \omega \\
\mathbf{B} \triangleq \frac{1}{2 \pi} \int_{0}^{2 \pi} \mathbf{d}\left(e^{j \omega}\right) \mathbf{H}\left(e^{j \omega}\right) \mathbf{S}_{\mathbf{x x}}\left(e^{j \omega}\right) d \omega
\end{gathered}
$$

Using the trick of completing the square, the optimal choice of $\overline{\mathbf{f}}_{c}$ which minimizes $\xi$ in (4) is given by [2],

$$
\overline{\mathbf{f}}_{c, \mathrm{opt}}=\mathbf{B}^{\dagger} \mathbf{A}^{\#}
$$

where $\mathbf{P}^{\#}$ denotes the Moore-Penrose pseudoinverse of the matrix $\mathbf{P}[2]$. In this case, the optimal error becomes,

$$
\xi_{\mathrm{opt}}=\operatorname{Tr}\left[\mathbf{R}_{\mathbf{x x}}(0)-\mathbf{B}^{\dagger} \mathbf{A}^{\#} \mathbf{B}\right]
$$

With the optimal choice of $\overline{\mathbf{f}}_{c}$ given in (5), the corresponding optimal synthesis bank $\mathbf{F}(z)$ can be computed using $\mathbf{F}(z)=\overline{\mathbf{f}}_{c} \mathbf{d}(z)$.

\subsection{Optimal Analysis Bank For Fixed Synthesis Bank}

Suppose now that the synthesis bank $\mathbf{F}(z)$ is fixed and that the analysis bank $\mathbf{H}(z)$ is FIR of length $N_{h}$ and of the form,

$$
\mathbf{H}(z)=z^{Q} \mathbf{H}_{c}(z)
$$

where $Q$ is an advance parameter and $\mathbf{H}_{c}(z)$ is a causal FIR system of the form,

$$
\mathbf{H}_{c}(z)=\sum_{n=0}^{N_{h}-1} \mathbf{h}_{c}(n) z^{-n}
$$

Note that the impulse response $\mathbf{h}_{c}(n)$ is an $L \times M$ sequence and that there are $N_{h}$ such matrix coefficients. Hence, $\mathbf{H}(z)$ is characterized by a total of $L M N_{h}$ degrees of freedom with the FIR 
constraint in effect. In order to minimize $\xi$ from (2) in this case, we need to group all of these degrees of freedom together, which can be done through the use of the vec operator [2]. For simplicity, define the $L M \times 1$ column vectors $\overline{\mathbf{h}}_{n}$ as follows.

$$
\overline{\mathbf{h}}_{n} \triangleq \operatorname{vec}\left(\mathbf{h}_{c}(n)\right), 0 \leq n \leq N_{h}-1
$$

Furthermore, define the $L M N_{h} \times 1$ vector $\overline{\mathbf{h}}$, the $L M \times 1$ vector $\mathbf{v}(z)$ and the $L M N_{h} \times L M$ advance matrix $\mathbf{a}(z)$ as follows.

$$
\begin{aligned}
\overline{\mathbf{h}} & \triangleq\left[\begin{array}{cccc}
\overline{\mathbf{h}}_{0}^{T} & \overline{\mathbf{h}}_{1}^{T} & \cdots & \overline{\mathbf{h}}_{N_{h}-1}^{T}
\end{array}\right]^{T} \\
\mathbf{v}(z) & \triangleq \operatorname{vec}\left(\widetilde{\mathbf{F}}(z) \mathbf{S}_{\mathbf{x x}}(z)\right) \\
\mathbf{a}(z) & \triangleq\left[\begin{array}{lllll}
z^{-Q} \mathbf{I}_{L M} & z^{1-Q} \mathbf{I}_{L M} & \cdots & z^{N_{h}-1-Q} \mathbf{I}_{L M}
\end{array}\right]^{T}
\end{aligned}
$$

Note that $\overline{\mathbf{h}}$ contains all of the degrees of freedom of $\mathbf{H}(z)$ here. Exploiting the following properties of the trace and vec operators [2],

$$
\begin{aligned}
\operatorname{Tr}\left[\mathbf{A}^{\dagger} \mathbf{B}\right] & =(\operatorname{vec}(\mathbf{A}))^{\dagger} \operatorname{vec}(\mathbf{B}) \\
\operatorname{vec}(\mathbf{A X B}) & =\left(\mathbf{B}^{T} \otimes \mathbf{A}\right) \operatorname{vec}(\mathbf{X})
\end{aligned}
$$

where $\otimes$ denotes the Kronecker product operator [2], then from (3), we have, after some algebraic manipulation,

$$
\begin{aligned}
\operatorname{Tr}\left[\mathbf{S}_{\mathbf{e e}}(z)\right]= & \operatorname{Tr}\left[\mathbf{S}_{\mathbf{x x}}(z)\right]-\overline{\mathbf{h}}^{\dagger}(\mathbf{a}(z) \mathbf{v}(z))-(\widetilde{\mathbf{v}}(z) \widetilde{\mathbf{a}}(z)) \overline{\mathbf{h}} \\
& +\overline{\mathbf{h}}^{\dagger}\left(\mathbf{a}(z)\left(\mathbf{S}_{\mathbf{x x}}^{T}(z) \otimes \widetilde{\mathbf{F}}(z) \mathbf{F}(z)\right) \widetilde{\mathbf{a}}(z)\right) \overline{\mathbf{h}}
\end{aligned}
$$

Using (6) in (2), it can be shown that we have,

$$
\xi=\operatorname{Tr}\left[\mathbf{R}_{\mathbf{x x}}(0)\right]-\overline{\mathbf{h}}^{\dagger} \mathbf{g}-\mathbf{g}^{\dagger} \overline{\mathbf{h}}+\overline{\mathbf{h}}^{\dagger} \mathbf{C} \overline{\mathbf{h}}
$$

where the $L M N_{h} \times 1$ vector $\mathbf{g}$ and $L M N_{h} \times L M N_{h}$ matrix $\mathbf{C}$ are defined as follows,

$$
\begin{gathered}
\mathbf{g} \triangleq \frac{1}{2 \pi} \int_{0}^{2 \pi} \mathbf{a}\left(e^{j \omega}\right) \mathbf{v}\left(e^{j \omega}\right) d \omega \\
\mathbf{C} \triangleq \frac{1}{2 \pi} \int_{0}^{2 \pi} \mathbf{a}\left(e^{j \omega}\right)\left(\mathbf{S}_{\mathbf{x} \mathbf{x}}^{T}\left(e^{j \omega}\right) \otimes \mathbf{F}^{\dagger}\left(e^{j \omega}\right) \mathbf{F}\left(e^{j \omega}\right)\right) \mathbf{a}^{\dagger}\left(e^{j \omega}\right) d \omega
\end{gathered}
$$

As before, using the trick of completing the square on $\xi$ from (7), the optimal choice of $\overline{\mathbf{h}}$ which minimizes $\xi$ is given by [2],

$$
\overline{\mathbf{h}}_{\text {opt }}=\mathbf{C}^{\#} \mathbf{g}
$$

In this case, the optimal error becomes,

$$
\xi_{\text {opt }}=\operatorname{Tr}\left[\mathbf{R}_{\mathbf{x x}}(0)\right]-\mathbf{g}^{\dagger} \mathbf{C}^{\#} \mathbf{g}
$$

To summarize the main points of this section, we have derived closed form expressions for the optimal synthesis/analysis bank subject to an FIR constraint under the assumption that the corresponding analysis/synthesis bank is fixed. The notion of alternately fixing the analysis/synthesis bank and optimizing the synthesis/analysis bank will form the basis of an iterative algorithm used to obtain an optimal analysis/synthesis pair. It should be noted that this algorithm is not guaranteed to be globally optimal, since it will depend on the initialization used. However, the error at each iteration is guaranteed to not increase since the algorithm is greedy (i.e. at each stage in the iteration, the optimal analysis/synthesis bank is computed). We now proceed to formally state the iterative filter bank optimization algorithm.

\section{ITERATIVE ANALYSIS/SYNTHESIS FILTER BANK OPTIMIZATION ALGORITHM}

In what follows, let $\mathbf{F}_{k}(z), \mathbf{H}_{k}(z)$, and $\xi_{k}$ denote, respectively, the synthesis bank, analysis bank, and reconstruction error at the $k$-th iteration for $k \geq 0$. Then, the iterative filter bank optimization algorithm is as follows.

\section{Initialization:}

1. Select a set of prescribed values for the desired filter bank parameters $L, M, P, N_{f}, Q$, and $N_{h}$.

2. Choose an initial synthesis bank $\mathbf{F}_{0}(z)$.

3. Compute the corresponding optimal analysis bank $\mathbf{H}_{0}(z)$ and filter bank error $\xi_{0}$ using (8) and (9), respectively.

Iteration: For $k \geq 1$, do the following.

1. With a fixed analysis bank $\mathbf{H}_{k-1}(z)$, compute the optimal synthesis bank $\mathbf{F}_{k}(z)$ using (5).

2. With a fixed synthesis bank $\mathbf{F}_{k}(z)$, compute the optimal analysis bank $\mathbf{H}_{k}(z)$ and corresponding reconstruction error $\xi_{k}$ using (8) and (9), respectively.

3. Increment $k$ by 1 and return to Step 1 .

As stated earlier, since this algorithm is greedy, the error $\xi_{k}$ is guaranteed to be a monotonic nonincreasing function of the iteration index $k$. Since the error is always lower bounded by zero (i.e. $\xi_{k} \geq 0$ ), $\xi_{k}$ is also guaranteed to have a limit as $k \rightarrow \infty$ [7]. Simulation results provided here verify this monotonic and limiting behavior. In particular, it will be seen that the error appears to quickly converge to its limit after only a few iterations.

\section{SIMULATION RESULTS}

To test the proposed iterative optimization algorithm, we chose the input process $x(n)$ to be a WSS autoregressive process of order 4 $(\mathrm{AR}(4))$ whose power spectrum $S_{x x}\left(e^{j \omega}\right)$ is plotted in Fig. 5. For the filter bank parameters, we chose the following.

$$
L=1, M=3, P=0, N_{f}=7, Q=N_{h}-1, N_{h}=7
$$

In other words, we opted to design one subband of a three channel system in which the synthesis filter $F_{0}(z)$ is a causal FIR filter of length $M N_{f}=21$ and the analysis filter $H_{0}(z)$ is an anticausal FIR filter of length $M N_{h}=21$. Here, we chose the synthesis filter to be causal and the analysis filter to be anticausal and of the same length so as not introduce a temporal bias into the filter bank. For the initialization, the initial synthesis bank $\mathbf{F}_{0}(z)$ was chosen to be a random causal paraunitary system of order $\left(N_{f}-1\right)$ [7].

A plot of the reconstruction error $\xi_{k}$ as a function of the iteration index $k$ is shown in Fig. 2. The total number of iterations we used here was 20. Despite this small number, it can be seen that the error indeed is monotonic nonincreasing and appears to be approaching a limit. After the last iteration, the error was 2.0258 (i.e. $\left.\xi_{19}=2.0258\right)$. In contrast to this, for the unconstrained order orthonormal and biorthogonal solutions, the optimal error is 0.6539 . As we increase the order of the filters $\left(N_{f}\right.$ and $N_{h}$ ), the observed error comes closer to the optimal error in the unconstrained cases and may in fact surpass this error since we are not enforcing an orthonormal or biorthogonal constraint. When we ran the same simulations but increased $N_{f}$ and $N_{h}$ both to 8 , the observed reconstruction error after 20 iterations was found to be 2.0079 . 


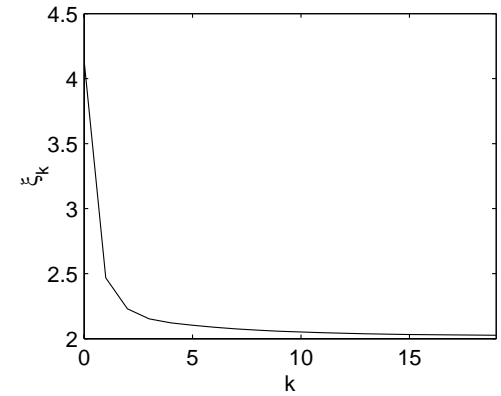

Fig. 2. Reconstruction error $\xi_{k}$ vs. the iteration index $k$.

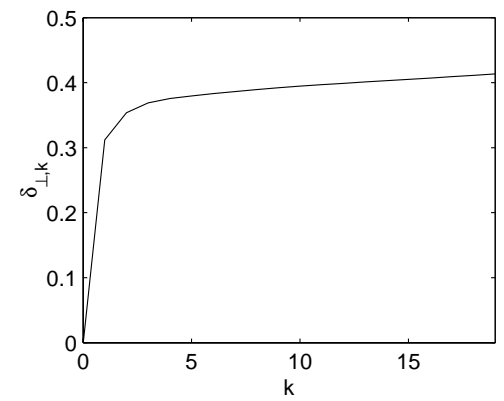

Fig. 3. Deviation from orthonormality $\delta_{\perp, k}$ vs. $k$.

In order to analyze the behavior of the solutions obtained with our algorithm, we opted to calculate the deviation of the observed solutions from orthonormality and biorthogonality. To measure the deviation from orthonormality, we considered the metric,

$$
\delta_{\perp, k} \triangleq \frac{1}{2 \pi} \int_{0}^{2 \pi}\left\|\mathbf{I}_{L}-\mathbf{F}_{k}^{\dagger}\left(e^{j \omega}\right) \mathbf{F}_{k}\left(e^{j \omega}\right)\right\|_{2}^{2} d \omega
$$

whereas to measure the deviation from biorthogonality, we used,

$$
\delta_{\mathrm{BIO}, k} \triangleq \frac{1}{2 \pi} \int_{0}^{2 \pi}\left\|\mathbf{I}_{L}-\mathbf{H}_{k}\left(e^{j \omega}\right) \mathbf{F}_{k}\left(e^{j \omega}\right)\right\|_{2}^{2} d \omega
$$

In Fig. 3 and Fig. 4, we have plotted, respectively, $\delta_{\perp, k}$ and $\delta_{\text {BIO }, k}$ as functions of $k$. From Fig. 3, it can be seen that the solution obtained deviates monotonically from orthonormality, whereas in Fig. 4, the solution fluctuates but appears approximately biorthogonal.

Finally, in Fig. 5, we have plotted the input spectrum $S_{x x}\left(e^{j \omega}\right)$ along with the magnitude responses of the analysis and synthesis filters $H_{0}(z)$ and $F_{0}(z)$. As can be seen, the filters appear to try to capture the significant parts of the input spectrum while suppressing the insignificant portions. This is consistent with the behavior of the optimal orthonormal and biorthogonal solutions which compact the energy of the input into the first few subbands $[8,9]$.

\section{CONCLUDING REMARKS}

An iterative algorithm for computing optimal FIR analysis/synthesis systems for partial reconstruction in overdecimated filter banks was proposed and shown to be useful in simulations. Future research includes deriving a similar algorithm for a more general rate-distortion type objective such as coding gain.

\section{REFERENCES}

[1] S. Akkarakaran and P. P. Vaidyanathan, "Filterbank optimization with convex objectives and the optimality of principal component forms," IEEE Trans. Signal Processing, 49(1):100-114, Jan. 2001.

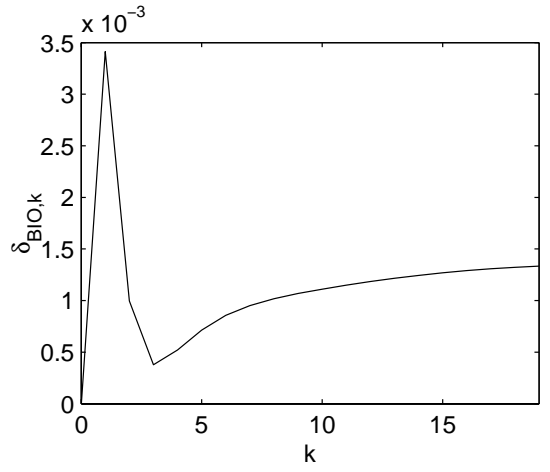

Fig. 4. Deviation from biorthogonality $\delta_{\mathrm{BIO}, k}$ vs. $k$.

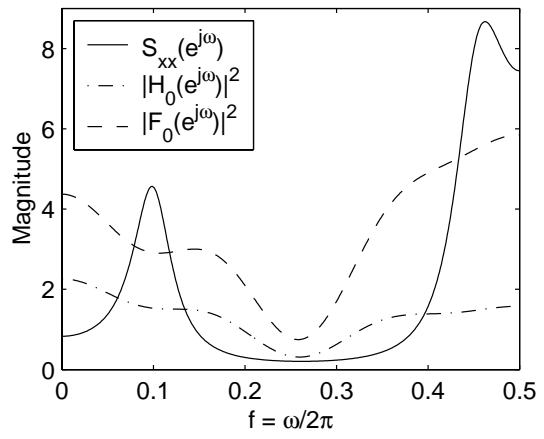

Fig. 5. Input power spectrum $S_{x x}\left(e^{j \omega}\right)$ and analysis/synthesis magnitude responses $\left|H_{0}\left(e^{j \omega}\right)\right|^{2}$ and $\left|F_{0}\left(e^{j \omega}\right)\right|^{2}$.

[2] T. K. Moon and W. C. Stirling, Mathematical Methods and Algorithms for Signal Processing, Prentice-Hall, Inc., Upper Saddle River, NJ, 2000.

[3] P. Moulin and M. K. Mihçak, "Theory and design of signaladapted FIR paraunitary filter banks," IEEE Trans. Signal Processing, 46(4):920-929, Apr. 1998.

[4] P. Moulin, M. Anitescu, and K. Ramchandran, "Theory of rate-distortion-optimal, constrained filterbanks-application to IIR and FIR biorthogonal designs," IEEE Trans. Signal Processing, 48(4):1120-1132, Apr. 2000.

[5] M. K. Tsatsanis and G. B. Giannakis, "Principal component filter banks for optimal multiresolution analysis," IEEE Trans. Signal Processing, 43(8):1766-1777, Aug. 1995.

[6] J. Tuqan and P. P. Vaidyanathan, "Statistically optimum preand postfiltering in quantization," IEEE Trans. Circuits Syst. II, 44(1):1015-1031, Dec. 1997.

[7] P. P. Vaidyanathan, Multirate Systems and Filter Banks, Prentice-Hall, Inc., Englewood Cliffs, NJ, 1993.

[8] P. P. Vaidyanathan, "Theory of optimal orthonormal subband coders," IEEE Trans. Signal Processing, 46(6):1528-1543, Jun. 1998.

[9] P. P. Vaidyanathan and A. Kıraç, "Results on optimal biorthogonal filter banks," IEEE Trans. Circuits Syst. II, 45(8):932-947, Aug. 1998.

[10] B. Xuan and R. H. Bamberger, "FIR principal component filter banks," IEEE Trans. Signal Processing, 46(4):930-940, Apr. 1998. 